\title{
Neural mechanisms supporting the extraction of general knowledge across episodic memories
}

\author{
Carly C.G. Sweegers ${ }^{\mathrm{a}, \mathrm{b}, *}$, Atsuko Takashima ${ }^{\text {c,d }}$, Guillén Fernández ${ }^{\mathrm{c}, \mathrm{e}}$, Lucia M. Talamini a,b \\ a Department of Psychology, University of Amsterdam, Netherlands \\ b Cognitive Science Center Amsterdam, University of Amsterdam, Netherlands \\ c Donders Institute for Brain, Cognition and Behaviour, Radboud University Nijmegen, Netherlands \\ d Behavioural Science Institute, Radboud University Nijmegen, Netherlands \\ e Department of Cognitive Neuroscience, Radboud University Medical Centre, Netherlands
}

\section{A R T I C L E I N F O}

\section{Article history:}

Accepted 28 October 2013

Available online 9 November 2013

\section{Keywords:}

Episodic memory

Semantic memory

System-level consolidation

Hippocampus

fMRI

\begin{abstract}
A B S T R A C T
General knowledge acquisition entails the extraction of statistical regularities from the environment. At high levels of complexity, this may involve the extraction, and consolidation, of associative regularities across event memories. The underlying neural mechanisms would likely involve a hippocampo-neocortical dialog, as proposed previously for system-level consolidation. To test these hypotheses, we assessed possible differences in consolidation between associative memories containing cross-episodic regularities and unique associative memories. Subjects learned face-location associations, half of which responded to complex regularities regarding the combination of facial features and locations, whereas the other half did not. Importantly, regularities could only be extracted over hippocampus-encoded, associative aspects of the items. Memory was assessed both immediately after encoding and $48 \mathrm{~h}$ later, under fMRI acquisition. Our results suggest that processes related to system-level reorganization occur preferentially for regular associations across episodes. Moreover, the buildup of general knowledge regarding regular associations appears to involve the coordinated activity of the hippocampus and mediofrontal regions. The putative cross-talk between these two regions might support a mechanism for regularity extraction. These findings suggest that the consolidation of cross-episodic regularities may be a key mechanism underlying general knowledge acquisition.
\end{abstract}

(C) 2013 Elsevier Inc. All rights reserved.

\section{Introduction}

The brain stores information about the statistical properties of the environment at all levels of complexity. This entails the extraction of regularities over spatially and temporally discontinuous events to form perceptual categories, sensorimotor routines and more complex forms of concepts and relations. The resulting, highly structured, information system is termed 'semantic memory' (Patterson et al., 2007; Tulving, 1985) and is thought to depend on networks comprising widespread cortical regions (Binder et al., 2009; Martin and Chao, 2001). Various observations suggest that the formation of semantic memories depends in large part on episodic memory (Moscovitch et al., 2005; Rosenbaum et al., 2001; but see Gardiner et al., 2008 for an opposite viewpoint) and an intact hippocampus (Bayley and Squire, 2005; Hayman et al., 1993; Manns et al., 2003). Episodic memory refers to memory for events and situations, organized in an autobiographical stream, and rich in contextual information (Tulving, 1983). This type of memory depends on the hippocampus to quickly encode the spatial and temporal relations between event components represented in

\footnotetext{
* Corresponding author at: Weesperplein 4, 1018 XA Amsterdam, Netherlands. E-mail address: sweegers@gmail.com (C.C.G. Sweegers).
}

distributed cortical regions (Burgess et al., 2002; Lisman et al., 2005; Meeter et al., 2004; Nadel and Moscovitch, 1997; Squire, 1992). The mechanisms underlying semantic memory formation may therefore involve some form of hippocampo-neocortical dialog, whereby only certain aspects of the original episodes are recoded to hippocampusindependent cortical representations.

Hippocampal-neocortical interactions have previously been shown to underlie system-level consolidation, the process through which hippocampus-dependent memories, over time, acquire a more cortically based, and more stable representation. Here, we propose that one principle governing this process relates to the extraction of regular associations over episodes. Indeed, memory representations reflecting such regularities may be consolidated preferentially at the expense of those reflecting incidental associations. This implies that different components of episodic memories may undergo different consolidation trajectories, depending on the stability of the associations they reflect and, therewith, the relevance of these associations in the long-term. In other words, memory traces may not be consolidated in their original form, but in a reduced state that reflects environmental regularities.

If extraction of regularities over hippocampus-dependent memory traces indeed occurs, it will likely involve the contemporaneous (re) activation of multiple episodic memories and detection of associative 
overlap. An area that may play a central role in such processing is the prefrontal cortex (PFC). Its well-known role in memory function relates to the organization of information to be memorized and the use of such organization in retrieval strategies (Blumenfeld and Ranganath, 2007; Otani, 2004). While in most pertaining experiments these functions regarded object categorization, the prefrontal cortex might play a similar role in the organization of episodic memories according to associative similarities. However, in the latter case, the necessary reactivation of stored episodic memory traces would plausibly require interplay of the prefrontal cortex with the hippocampus. The medial prefrontal cortex ( $\mathrm{mPFC}$ ) might be particularly important as lesions to this area impair category formation (Drewe, 1974) and shifting between response rules (Birrell and Brown, 2000). Moreover, since the MPFC receives monosynaptic input from the hippocampus, a direct portal for hippocampo-prefrontal communication is available (Swanson, 1981; Thierry et al., 2000). In line with this notion, several studies implicate hippocampo-mPFC communication in rule and schema learning (Benchenane et al., 2010; Kumaran et al., 2009; van Kesteren et al., 2010), which also involve interactions of new associative input with already stored information.

In view of the above, we expect that hippocampus-mPFC interactions might play a major role in the extraction of regularities over episodes. We, furthermore, hypothesize that system-level consolidation will be particularly evident for associations containing regularities, as compared to arbitrary associations. To test these hypotheses we introduce a task that requires the learning of face-location associations and later retrieval of locations based on face cues. Half of the material responds to complex associative regularities regarding the combination of facial features and locations, whereas the other half of the faces is randomly assigned to one of the remaining locations. This creates 'rule-locations': locations that have a specific type of face associated with it, and 'no-rule locations': locations that can be paired with any type of face. During encoding, the regularities associated with a given location can only be extracted across multiple face-location items that are presented interleaved with faces from other locations. This means that the build-up of hippocampus-dependent representations of individual face-location associations is a necessary step towards regularity extraction.

Memory for the location of faces is tested shortly after encoding and 48 h later, while brain activity is monitored through functional magnetic resonance imaging (fMRI). We expect that the neural networks underlying retrieval of rule-based and non-rule based items will be similar shortly after learning, but will differentiate over time, consequent to the extraction and preferential consolidation of cross-episodic regularities.

\section{Materials and methods}

\section{Subjects}

Thirty-one subjects (five males, mean age \pm SD: $20.76 \pm 2.71$ ) gave written informed consent and received either course credits or a financial compensation for participation in this experiment, which was approved by the local ethics committee. Six were excluded: two for not reaching the pre-set number of trials (10) in a given condition needed for the fMRI analysis, two for expressing insufficient explicit rule knowledge as measured with an exit questionnaire, ${ }^{1}$ one due to joystick malfunctioning during scanning and finally one based on an outlier analysis on retrieval performance $(z$-score $<-2.5)$. The final group therefore consisted of 25 subjects.

\footnotetext{
${ }^{1}$ In order to make sure that the rule trials reflect trials for which subjects actually made use of rules, subjects that did not pick at least 2 out of 3 correct rule locations in the closedformat version of the rules questionnaire were excluded from the analysis.
}

Stimuli

Seventy-two grayscale pictures of emotionally neutral faces were created using Faces ${ }^{\mathrm{TM}}$ software (IQ Biometrix, 2003). Each face had several non-critical features. However, three critical features occurred in each face: faces were either 1) young adult or aged; 2) slender or stout and had either 3 ) headwear (caps, hats or headbands) or no headwear. For 6 out of the 8 possible 3-way combinations of these features 12 faces were created. Each of these face categories had one unique combination of two features (e.g. headwear and slender face) that did not occur in other categories. The critical facial features could come in various forms (e.g. different types of headwear, and wrinkle patterns), contributing to the perceptual distinctiveness of the faces (see Supplementary materials Table 1, for a full description of the six face categories).

Each face was coupled to one of six screen locations in order to create face-location associations (12 faces to each location). Three locations were appointed as rule-locations, meaning that all 12 faces associated to that location belonged to the same category. The other three locations were no-rule-locations and the faces in the three remaining categories were randomly assigned to these locations. Thus, half of the associations to be learned responded to regularities governing face placements, whereas the other half did not (see Fig. 1 for an illustration of the experimental design and the Supplementary materials for an elaboration of the task rationale). Finally, the positions of the rule/no-rule locations, as well as the categories associated with the rule/no-rule locations were counterbalanced over subjects.

\section{Procedure}

Subjects were informed that the goal of the task was to learn all 72 face-location associations. They were told that for some locations they could learn rules, made up of the combinations of physical facial features, which could help them place the faces at that location. Subjects were not informed which locations or what physical features were relevant for regularity extraction. Therefore, the initial learning strategy was the same for rule and no-rule faces. The learning phase consisted of four encoding-retrieval cycles. During an encoding block, each of the 72 faces popped up over a mid-screen fixation cross and moved to one of the six locations that were organized hexagonally around the fixation cross. Immediately after each encoding block, a retrieval block followed in which subjects were instructed to indicate the correct location of each face. Subjects used a joystick to move the cursor from the fixation cross to the selected location and confirmed their choice with a button press. In the first three cycles, subjects received feedback on each placement: if the correct location was chosen, a green circle appeared at the correct location, and the face moved to that location; if an incorrect location was chosen, a red circle appeared at the incorrect location, followed by a green circle at the correct location. Subsequently, the subject had to make a movement to the correct location, after which the face moved to that location. In the fourth retrieval block, no feedback was given, but subjects had to indicate their response confidence on a five-point scale $(1=$ unsure to $5=$ sure). A short break was given after each cycle, with a longer break (10 min) after the second cycle, during which subjects made puzzles. The order of the faces was randomized over blocks and over subjects, with the proviso that the same set of faces always appeared in either the first or the second half of each block.

Thirty minutes after the end of the learning phase, subjects entered the first fMRI session (recent condition). The second fMRI session occurred $48 \mathrm{~h}$ after the start of the learning phase (remote condition). During both scan sessions, subjects performed a cued recall test for face-location memory under fMRI data acquisition. All 72 faces served as cues and were presented on the fixation cross, in random order. Each face, surrounded by the six location probes, stayed on-screen for $4 \mathrm{~s}$. Subjects were instructed to indicate the correct location by a 
joystick movement, using their right hand, and to confirm their choice with a button press using their left index finger. If the correct location was chosen within the $4 \mathrm{~s}$ time frame, the trial was considered correct. No feedback was given. After a jittered interval of 1-2 s, subjects were additionally instructed to rate the confidence of their response. In case they did not remember the location, or failed to pick a location within the $4 \mathrm{~s}$ time limit, they were instructed to indicate the lowest confidence rating of 1 . Each trial was followed by a jittered inter-trial interval of 3-7 s during which the fixation cross was presented at the center of the screen.

At the end of the first retrieval session, a structural magnetic resonance imaging (MRI) scan was obtained. After the second retrieval session, subjects performed a localizer task in the scanner, in order to identify subject-specific face-processing areas (bilateral fusiform face area $[F F A])$. Subjects performed a one-back working memory task while they were presented with blocks (13 images each) containing sequences of either faces, scrambled images or houses. The task was to press the button whenever they saw the same picture twice in a row.

At the end of the second retrieval session subjects filled out a questionnaire designed to assess their explicit rule knowledge. Subjects were asked at what time point during the experiment they had started to get a notion of any regularity and also up to what time point they had kept on updating these notions. Next, subjects were informed that there had been three rule-locations, and were asked to indicate the three possible positions thereof (forced choice). Moreover, for each of the three rule-locations they could indicate whether they thought the rules involved the face's gender and/or the presence or absence of one or more of the following five features: headwear, wrinkles, stoutness, glasses and mole. Points were given for each correctly chosen location (max. 3), as well as for each correct physical feature that was part of the rule (max. 3 per location). The total maximum score of this questionnaire, summed over location and feature hits, was 12 points.

\section{MRI data acquisition}

T2*-weighted images covering the whole brain were acquired (31 axial slices, ascending slice acquisition, repetition time $(\mathrm{TR})=$ $2280 \mathrm{~ms}$, echo time $(\mathrm{TE})=35 \mathrm{~ms}, 90^{\circ}$ flip angle, matrix $=64 \times 64$, slice thickness: $3.5 \mathrm{~mm}$, slice gap $=0.35 \mathrm{~mm}$ and field of view (FOV): $212 \mathrm{~mm}$ ) using an echo-planar imaging (EPI) sequence on a $1.5 \mathrm{~T}$ Siemens Magnetom Avanto system. For structural MRI, T1-weighted images were acquired using a magnetization-prepared, rapid-acquisition gradient echo sequence (176 sagittal slices, TR $=2250 \mathrm{~ms}$, TE $=$ $2.95 \mathrm{~ms}, 15^{\circ}$ flip angle, matrix $=256 \times 256$, slice thickness: $1.0 \mathrm{~mm}$, FOV: $256 \mathrm{~mm})$.

\section{MRI data analysis}

Image preprocessing and statistical analysis were performed using SPM8 (www.fil.ion.ucl.ac.uk). The first five volumes of each participant's functional EPI data were discarded to allow for T1 equilibration. The EPI images were realigned to the first functional volume and the subject mean was co-registered with the corresponding structural MRI using mutual information optimization. Structural MRI data were segmented and probabilistic maps of gray matter and white matter were created for each subject. Both functional and structural scans were spatially normalized and transformed into a common Montreal Neurological Institute space (resampled at voxel size $2 \times 2 \times 2 \mathrm{~mm}$ ), as defined by the SPM8 T1.nii template, as well as spatially filtered by convolving the functional images with an isotropic three-dimensional (3D) Gaussian kernel ( $8 \mathrm{~mm}$ full width at half maximum).

The fMRI data were analyzed statistically using the general linear model (GLM) and statistical parametric mapping. The hemodynamic response to each stimulus event was modeled as a canonical hemodynamic response function (HRF) along with its temporal derivatives provided by SPM8. Each event was time-locked to the presentation of the face. The design matrix included six head motion regressors (translations, rotations) to account for any residual movement-related effects. A high-pass filter was implemented using a cut-off period of $128 \mathrm{~s}$ to remove low frequency effects from the time series. The following conditions of interest were modeled: correct responses to recent rule faces, correct responses to recent no-rule faces, correct responses to remote rule faces, correct responses to remote no-rule faces and finally all incorrect trials. Even at low response confidence, there might be implicit usage of built-up rules, so trials with all confidence levels were included in the corresponding regressors. Relevant contrast parameter images were created for each condition to the implicit baseline (mean signal during unmodeled periods). These images were generated for each subject and were subjected to a full factorial analysis, embedded in SPM8, with TIME (recent/remote) and RULE (rule/no-rule) as independent variables. In this factorial analysis, independence and equal variance between the conditions were not assumed. Subject covariates were added to account for inter-individual differences.

\section{Experimental design}
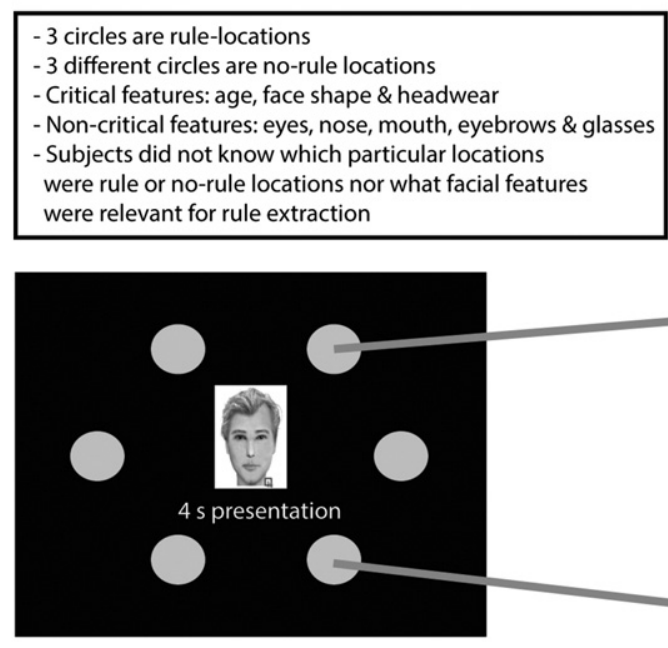
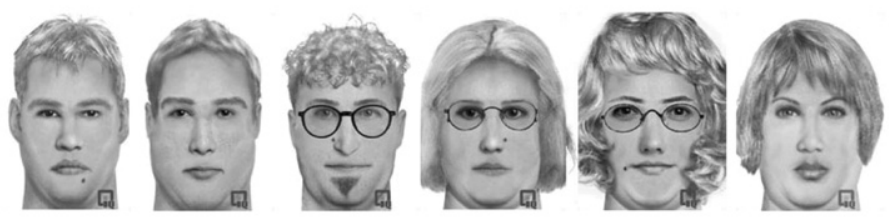

Rule location: critical 3-way combination: Young, stout and no headwear
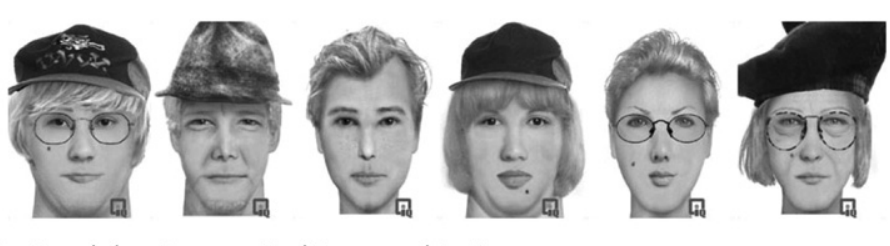

No-rule location: no critical 3-way combination: young/aged \& slender/stout \& headwear/no headwear

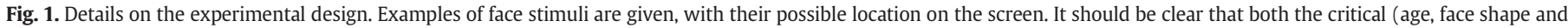

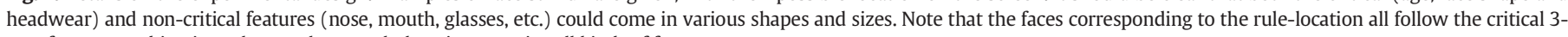
way feature combination whereas the no-rule-location contains all kinds of faces. 
To probe changes in functional connectivity between conditions of interest, we adopted a psychophysiological interaction (PPI) analysis (Friston et al., 1997) embedded in SPM8. A PPI analysis investigates task-related changes in co-activation between a seed region and all voxels outside this seed region. Several functionally defined seed regions were chosen to investigate functional connectivity: the FFA, the hippocampus, and finally a region encompassing the anterior cingulate cortex (ACC) and medial prefrontal cortex ( $\mathrm{MPFC}$ ), which resulted from the PPI analysis with the hippocampus as seed region (see the 'Results' section).

The FFA was chosen because the face served as the cue to retrieve the corresponding location and the FFA has been consistently shown to be active during facial processing tasks (Gauthier et al., 2000; Grill-Spector et al., 2004; Kanwisher et al., 1997). The FFA seed region was defined per subject based on the face-house contrast in the localizer task. The localizer scans were processed using procedures similar to those for the retrieval runs. In short, hemodynamic responses were modeled using a statistical parametric map, in which blocks of $18 \mathrm{~s}$ on/off were modeled as boxcar functions convolved with a hemodynamic response function. Individual movement regressors were again added to each first-level model. Due to betweensubject variability regarding the ease of detection of the FFA activity peak, the face-scrambled image contrast had to be used for a few subjects, instead of the more common face-house contrast. $10 \mathrm{~mm}$ spheres were drawn (bilaterally) surrounding the voxels showing maximal activity in the fusiform gyrus.

The hippocampal seed ROI was defined as follows: average activation peaks within the bilateral hippocampus were calculated for all correct trials combined against the baseline. For each hemisphere, the peak voxel was detected and two spheres of $10 \mathrm{~mm}$ diameter, surrounding these voxels (left $[-22,-28,-2]$ and right $[20,-30,0]$ ), composed a bilateral hippocampal ROI.

Finally, the ACC/mPFC seed, a $10 \mathrm{~mm}$ sphere surrounding the $[8,30$, 20 ] coordinate, resulted from the functional connectivity analysis with the hippocampus. The selection of the hippocampal and ACC/mPFC seeds is explained in more detail in the Results section.

For the PPI analyses, GLM's were constructed at the single subject level using three regressors: (1) the deconvolved signal from the seed region (defined by the first eigenvariate extracted from the signals of all voxels within the seed region, which in the case of the FFA and hippocampus is bilateral), (2) trial onset for the condition of interest, convolved with the HRF, and (3) the interaction term between the first and second regressors. In addition, six movement-related regressors were included in the GLM. Conditions of interest were: correct responses to recent rule faces, correct responses to recent no-rule faces, correct responses to remote rule faces and correct responses to remote no-rule faces. For each of these conditions, a separate GLM was constructed. Contrast images of the interaction regressor were used as input to the second level 2 (TIME) $\times 2$ (RULE) full factorial analysis.

When a planned PPI analysis revealed a TIME $\times$ RULE interaction, additional statistics were performed to unravel 1) possible differences between the rule and no-rule conditions at the recent and/or remote time point and 2) increases or decreases for the rule and/or no-rule condition across time. Specifically, for the region of interest (ROI) showing an interaction effect, beta weights, reflecting the relative functional connectivity strength for each of the four conditions (recent rule, recent norule, remote rule, and remote no-rule) against implicit baseline, were extracted. Mean beta weights, per subject and per condition, were calculated using the Marsbar toolbox (http://marsbar.sourceforge.net/ projects/marsbar) and used as input for post-hoc statistics (SPSS 18.0). When the PPI analysis revealed a significant difference at a specific time point only, beta values were similarly extracted but merely served visualization purposes, as additional statistics on these values would result in inflated p-values.

Results of all full factorial analyses, regarding either activity or connectivity data, were initially thresholded at $\mathrm{p}<0.001$ (voxel-level, uncorrected). Further, cluster-size statistics were used as test statistics, applying a threshold of $\mathrm{p}<0.05$ (family-wise error corrected, based on random field theory; Hayasaka and Nichols, 2003) for the whole-brain search. For specific ROIs, small volume corrections (SVCs) were performed using anatomical masks embedded in SPM8. The SVC method, as implemented in SPM8, allows the results to be corrected for multiple comparisons within a previously defined ROI.

\section{Results}

\section{Behavioral results}

At the end of the encoding phase, correct retrieval of face-location associations was well above chance level (mean \pm SD: $86.2 \pm 11.9 \%$; chance level 16.7\%). The occurrence of regularities boosted performance, as apparent from higher accuracy $(\mathrm{t}(24)=3.56, \mathrm{p}=0.002)$, as well as higher confidence $(\mathrm{t}(24)=2.98, \mathrm{p}=0.006)$ and shorter reaction times $(\mathrm{RTs})(\mathrm{t}(24)=-2.65, \mathrm{p}=0.014)$ for correct responses in the rule condition compared to the no-rule condition (Table 1).

Memory performance during the two retrieval scans was analyzed through repeated measures ANOVA's with within-subject factors TIME (recent/remote) and RULE (rule/no-rule). Separate analyses were performed for accuracy, confidence rating and RT. Main effects of TIME were found on all three measures, showing that, with time, subjects' responses became less accurate $(\mathrm{F}(1,24)=18.91, \mathrm{p}<0.0005)$, slower $(\mathrm{F}(1,24)=5.91, \mathrm{p}=0.023)$ and less confident $(\mathrm{F}(1,24)=19.56$, $\mathrm{p}<0.0005)$. More importantly, main effects of RULE indicated that when regularities were present, subjects were on average more accurate $(F(1,24)=33.74, p<0.0005)$, faster $(F(1,24)=14.04$, $\mathrm{p}=0.001)$ and more confident $(\mathrm{F}(1,24)=4.92, \mathrm{p}=0.036)$. In addition, an interaction was found between TIME and RULE on accuracy scores $(F(1,24)=5.27, p=0.031)$, reflecting better memory retention in the rule condition (Fig. 2). Adding the accuracy scores at the end of encoding into the analysis $(3 \times 2$ repeated measures ANOVA) rendered this interaction effect even more pronounced $(\mathrm{F}(1,24)=13.77, \mathrm{p}<0.0005)$. Next, we carried out post-hoc t-tests to investigate behavioral differences at each retrieval point separately. At the recent time point, regularities positively influenced accuracy $(\mathrm{t}(24)=4.72, \mathrm{p}<0.0005)$, RTs $(\mathrm{t}(24)=-2.56, \mathrm{p}=0.017)$ and confidence ratings $(\mathrm{t}(24)=2.31, \mathrm{p}=0.030)$. At the remote time point, only the effects on accuracy $(\mathrm{t}(24)=6.21, \mathrm{p}<0.0005)$ and RTs $(\mathrm{t}(24)=-3.61, \mathrm{p}=0.001)$ were significant, with confidence ratings being only marginally influenced by regularities $(\mathrm{p}=0.073)$.

At the end of the second retrieval session, subjects' explicit knowledge of the regularities was measured using a questionnaire. On average, subjects scored $52 \%$ correct on this questionnaire. Separating the two contributions to this score revealed that subjects scored $81 \%$ correct on the locations and $42 \%$ on the specific facial features. This means that, on average, they were able to correctly identify 2.4 of the 3 rule locations and 1.3 out of 3 correct features per location. ${ }^{2}$ None of the subjects showed full knowledge of the regularities. Furthermore, on average subjects started to get a notion of the rules roughly halfway through the encoding session and continued updating their rule knowledge until the end of the first retrieval session in the scanner. These questionnaire results suggest that knowledge of the regularities remained incomplete until the end of the experiment. Since storage of unique face-location combinations was necessary for regularity extraction to occur, it is likely that retrieval performance for the rule-based associations relied on a combination of episodic memory and regularity knowledge during all sessions.

\footnotetext{
${ }^{2}$ An extra measure of explicit rule knowledge was calculated, focusing solely on the correctly recalled combination of features ( 2 out of 3 ) that were unique for each rule category. Here, the total maximum score, including location points, was 9 points. By this calculation, explicit rule knowledge was only slightly higher (56\%). The location score remained $81 \%$ and the specific facial feature knowledge improved slightly $(43 \%)$.
} 
Table 1

Behavioral results, at 3 time points: end of encoding, recent retrieval session and remote retrieval session.

\begin{tabular}{|c|c|c|c|c|c|c|}
\hline & \multicolumn{2}{|l|}{ Accuracy } & \multicolumn{2}{|l|}{ RTs } & \multicolumn{2}{|l|}{ Confidence } \\
\hline & Rule & No-rule & Rule & No-rule & Rule & No-rule \\
\hline End of encoding & $91.33(9.08) \%$ & $81.11(17.46) \%$ & $2111(431) \mathrm{ms}$ & 2308 (431) ms & $4.47(0.36)$ & $4.24(0.54)$ \\
\hline Recent retrieval & $88.11(10.53) \%$ & $73.33(20.91) \%$ & $2229(294) \mathrm{ms}$ & $2324(250) \mathrm{ms}$ & $4.49(0.37)$ & $4.32(0.55)$ \\
\hline Remote retrieval & $82.56(15.74) \%$ & $62.89(20.79) \%$ & $2293(241) \mathrm{ms}$ & 2446 (279) ms & $4.27(0.42)$ & $4.12(0.59)$ \\
\hline
\end{tabular}

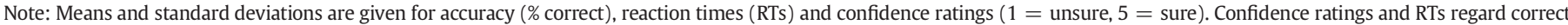
responses.

\section{Imaging results: activity}

The activation pattern related to successful retrieval of face-location associations (Fig. 3) (all correct trials against implicit baseline) is in close accord with previous findings using a similar task (Takashima et al., 2007, 2009). It encompasses areas coding for the face stimuli (ventral stream including the bilateral FFA, Gauthier et al., 2000; GrillSpector et al., 2004; Kanwisher et al., 1997) and the locations of these stimuli (dorsal stream including the posterior parietal cortex, Burgess et al., 2001; Kesner, 2009; Sack, 2009), as well as areas reflecting motor preparation for the joystick movement to the target location (supplementary motor cortex).

Activity data was analyzed through full factorial analysis, including factors TIME (recent/remote) and RULE (rule/no-rule). Main effects of TIME (collapsed over rule and no-rule conditions) were, first of all, found in a posterior region of the left hippocampus (peak [-22, -40 , 6], SVC), where activity decreased with the passage of time. Conversely, several regions showed a significant increase in activity over time: large clusters in the bilateral inferior frontal gyri, a large cluster including the right angular and middle occipital gyrus, a large cluster including the left inferior parietal lobe and the left middle temporal gyrus, and finally a cluster including the left fusiform and inferior temporal gyrus. No main effects were found for RULE, nor did we find any interactions between TIME and RULE in terms of retrieval-related activity. In the Supplementary data, detailed information on peak coordinates and cluster sizes can be found (Tables S2-S5). For an in-depth discussion of the general retrieval network, see our previous paper (Takashima et al., 2009).

\section{Imaging results: functional connectivity}

The functional connectivity correlates of regularity learning were assessed through several PPI analyses (Friston et al., 1997). Firstly, the bilateral FFA was used as seed region. The seed region was defined in each subject, based on regional activation in a localizer task, in order

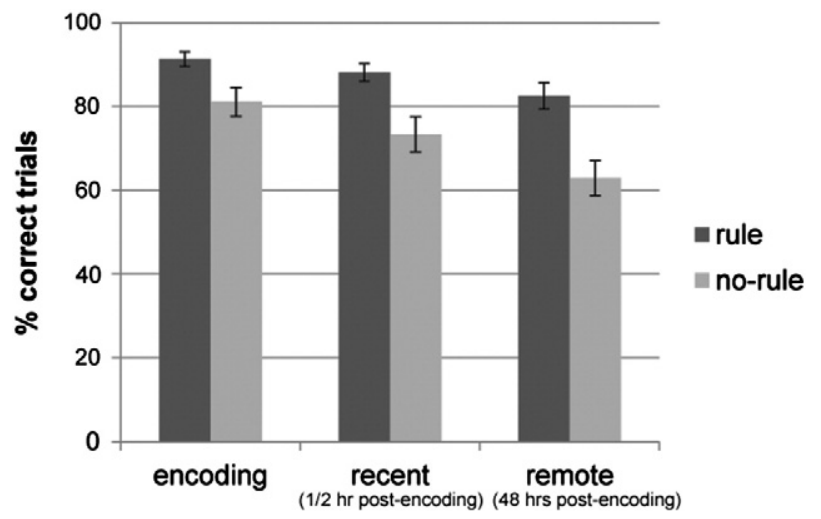

Fig. 2. Mean accuracy scores (given as \% correct trials) for the rule and the no-rule conditions, at the end of encoding and during the recent and remote retrieval sessions in the scanner. Error bars represent the standard error of the mean. to identify subject-specific face-processing areas. Full factorial analysis revealed a main effect of TIME, showing that connectivity between the bilateral FFA and the left hippocampus (peak $[-18,-14,-18]$, SVC) decreased over time. The main effect of RULE and the interaction between TIME and RULE were not significant. Since we expected differences between the rule and no-rule conditions to be specifically evident in the remote session, we performed a planned comparison between the remote rule and remote no-rule conditions. This revealed stronger functional connectivity for the remote rule condition, between the FFA and a cluster encompassing the right superior and inferior parietal lobes and angular gyrus (peak [32, -62, 46]; Fig. 4).

The results of this PPI thus support previous findings (Takashima et al., 2009), showing relative disengagement of the hippocampus from memory retrieval over time. Moreover, they suggest that, at the remote time point, cortico-cortical functional connectivity between areas coding for different components of an association (FFA and posterior parietal cortex; Takashima et al., 2007) is higher when retrieving rule-based as compared to non-rule based associations.

The second PPI analysis regarded functional connectivity with the hippocampus. Since all four conditions of interest elicited bilateral hippocampal activity with highly similar local maxima at the group level, the average left and right hemispheric peaks were calculated. Two spheres of $10 \mathrm{~mm}$ each, surrounding these peaks ([-22, -28, -2]

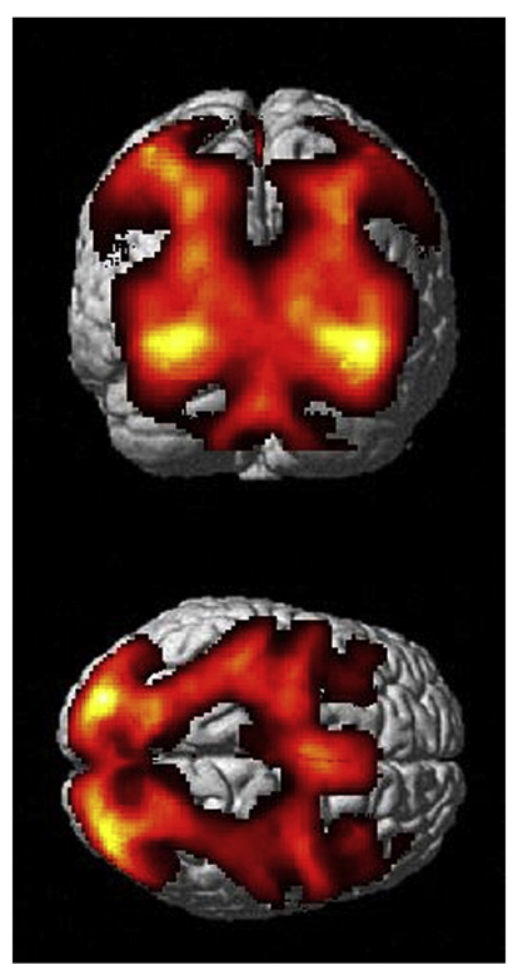

Fig. 3. Brain areas involved in the retrieval of correct face-location associations, collapsed over recent and remote time points, as well as rule and no-rule conditions (contrasted against baseline). 


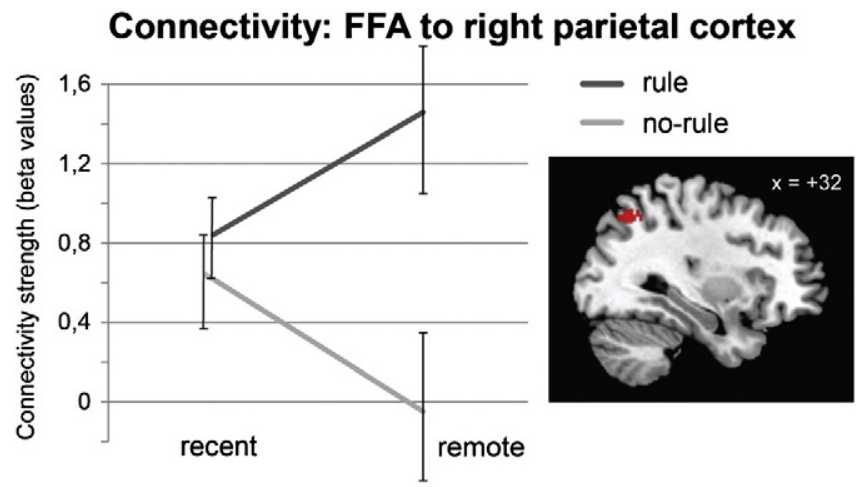

Fig. 4. Average beta weights per condition, reflecting connectivity strength between the FFA and a cluster in the right parietal cortex. The parietal cluster is shown in red in the sagittal brain cross section on the right, thresholded at $\mathrm{p}<.001$, uncorrected. This graph merely serves visualization purposes since statistics were not performed on these beta values.

and $[20,-30,0])$, composed a bilateral hippocampal ROI, which was used as a seed region in the PPI.

The full factorial analysis did not reveal any main effects of TIME or RULE. However, a significant interaction effect was found for connectivity between the hippocampus and a region encompassing the bilateral dorsal ACC and parts of the MPFC (peak $[8,30,20]$ in the medial aspect of the dorsolateral PFC, at the level of Brodmann area 9). To investigate this pattern further, the beta values reflecting the connectivity strength between the hippocampal ROI and this ACC/mPFC cluster were extracted, for each condition (Fig. 5). Post-hoc t-tests on these values revealed that connectivity differences between the rule and no-rule conditions were not significant in the recent condition, but connectivity was much higher for rule than no-rule items at the remote time point $(\mathrm{t}(24)=4.22, \mathrm{p}<0.0005)$. In addition, the rule faces elicited increasing connectivity between the hippocampus and ACC/mPFC across time $(\mathrm{t}(24)=-3.53, \mathrm{p}=0.002)$, whereas for no-rule faces connectivity decreased across time $(\mathrm{t}(24)=4.11, \mathrm{p}<0.005)$.

It thus appears that the ACC/mPFC plays a role in the retrieval of associations when this co-depends on knowledge of regularities. To examine this role, a third, exploratory, PPI analysis was done, with the ACC/mPFC cluster that resulted from the aforementioned PPI as a seed region. We were particularly interested in the TIME $\times$ RULE interaction, since it was from this interaction analysis, using the hippocampus as a seed, that the ACC/mPFC cluster appeared significant. The full factorial PPI analysis revealed a significant TIME $\times$ RULE

\section{Connectivity: hippocampus to ACC/mPFC}

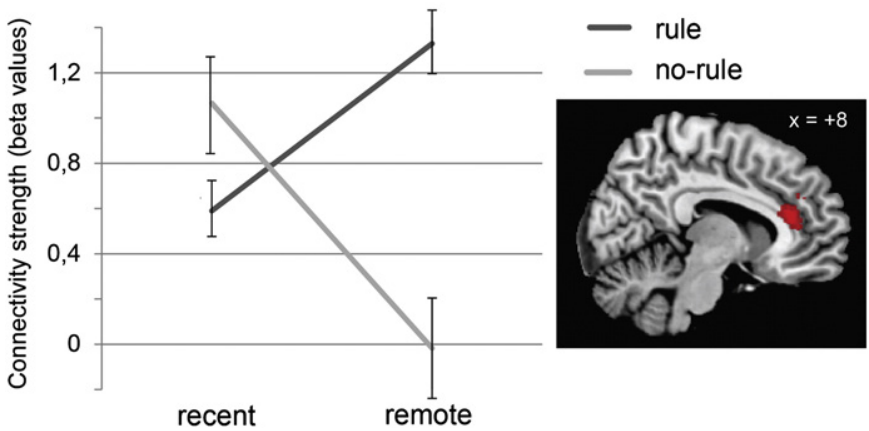

Fig. 5. Average beta weights per condition, reflecting connectivity strength between the hippocampus (HIP) and a cluster encompassing the anterior cingulate and medial prefrontal cortex (ACC/mPFC) - the latter is shown in red in the sagittal brain cross section on the right, thresholded at $\mathrm{p}<.001$, uncorrected. Error bars represent the standard error of the mean. interaction for coupling of the $\mathrm{ACC} / \mathrm{mPFC}$ with the left FFA (peak $[-30,-48,-18])$. Thus, over time the ACC/mPFC shows higher connectivity with facial processing areas when retrieving rulebased as compared to non-rule based associations. At a lower threshold $(\mathrm{p}<0.01)$, the TIME $\times$ RULE interaction also revealed bilateral regions in the hippocampus, but these clusters failed to reach our pre-set threshold at $\mathrm{p}<0.001$ voxel-level uncorrected.

\section{Discussion}

The ability to detect regularities among spatially and temporally discontinuous events is a key aspect of intelligent behavior. In the present study we investigated how the occurrence of regularities across face-location associations affected encoding, retention, and systemlevel changes in retrieval networks over $48 \mathrm{~h}$. A control condition consisted of similar associations that did not respond to any regularity. Our findings indicate, first, that regularities were indeed extracted, as evidenced by explicit knowledge thereof, as well as superior performance on the rule-based associations. Furthermore, although the neural networks supporting retrieval in the two conditions were very similar immediately after encoding, they differed at the remote time point. We will argue that this reflects the build-up of general knowledge regarding regularities in the material. Moreover, we will discuss the idea that the different retrieval networks at the remote time point might mark the start of diverging consolidation trajectories for the common and unique elements of episodes.

The behavioral benefits of cross-episodic regularities included enhanced performance during encoding and better retention of the pertaining associations. A plausible assumption holds that this is due to the higher endurance of those memory components reflecting the regularities in the material, compared to memory for the unique aspects of the associations.

Our findings at the brain level may be divided into general changes of retrieval networks over time, and differential changes according to the presence of regularities in the encoded material. With respect to the general changes, only one region, in the left posterior hippocampus, showed decreasing retrieval-related activity over time. Concomitantly, the functional connectivity between the FFA and left hippocampus also decreased. These results suggest a disengagement of the hippocampus from memory retrieval over time and replicate previous findings on system-level consolidation, using a similar face-place task, but without regularities (Takashima et al., 2009). A time-limited involvement of the hippocampus in event memory is in line with a large body of evidence, including studies in animals (Frankland et al., 2004; Maviel et al., 2004; Mishkin, 1978; Quillfeldt et al., 1996; Takehara et al., 2002; Zola-Morgan and Squire, 1985), humans (Bayley et al., 2006; Piefke, 2003; Scoville and Milner, 1957; Teng and Squire, 1999) and in silico (McClelland and Goddard, 1996; McClelland et al., 1995; Meeter and Murre 2005; Murre et al. 2006).

We also observed a relatively broad increase in retrieval-related activity over time. The increase is apparent in parts of the original retrieval network such as the bilateral inferior frontal gyri, but extends towards several association areas, i.e. the right angular gyrus and the left middle and inferior temporal gyri. The angular gyrus, a multimodal association area surrounding the parietal extension of the superior temporal sulcus, is anatomically interconnected with both the FFA and the posterior parietal area (Binder et al., 2009) and thus may function as a hub in their coupling. As such, the angular gyrus may take over some of the face-location binding initially subserved by the hippocampus. This notion should be considered with caution, however, as the spatial resolution of the adopted fMRI method limits reliable differentiation of the angular gyrus and adjacent regions in the posterior parietal area.

Somewhat surprisingly, we did not find any differences in activity measures between the rule and no-rule conditions. This suggests that very similar brain areas were involved in the retrieval of the locations in the rule and no-rule conditions. However, the communication 
between these areas differed significantly between the two conditions, reflecting differential retrieval processes for rule and no-rule items. In the paragraphs below we will discuss these functional connectivity differences.

First, functional connectivity was higher for rule-based than for nonrule based associations between the FFA and a cluster in the right posterior parietal cortex, at the remote time point. This cluster encompassed the inferior and superior parietal lobes and also the angular gyrus. The enhanced coupling between the FFA and parietal cortex might reflect the strengthening over time of cortico-cortical connections bypassing the hippocampus, and providing a more 'direct' route for recruitment of the location upon cueing with the face stimulus.

Notably, while our data suggest hippocampal disengagement from the face-location representations over time (to a similar extent in the rule and no-rule conditions), various studies suggest full hippocampal disengagement from episodic memories develops over weeks or longer (Brown, 2002; Maren et al., 1997; Squire et al., 1975; Talamini and Gorree, 2012). Thus, our findings may reflect partial rather than full hippocampal disengagement. A 'direct' route, as suggested above, may therefore, at least to some extent, act in addition to a remaining hippocampal connection. Accordingly, neural network models of system-level consolidation involve an intermediate consolidation state, in which cortico-cortical connectivity in neocortex is building up, while connections between the hippocampus and neocortex still bind the representation (McClelland and Goddard, 1996; McClelland et al., 1995; Meeter and Murre, 2005; Murre et al., 2006).

The above findings extend our previous work on system-level consolidation, which indicated increased cortico-cortical coupling for remotely acquired associations compared to recent ones (Takashima et al., 2009). Here, we show that such coupling is particularly evident for associations harboring regularities. We like to speculate that this reflects the start of a system-level consolidation process. System-level consolidation might, hence, be viewed as a competitive process, in which input reflecting relatively stable relations in the environment gains preferential access to consolidation mechanisms.

A second finding related to the existence of cross-episodic regularities regards the preferential build-up over time of functional connectivity between the hippocampus and a bilateral frontal cluster, spanning the dorsal ACC and extending into the MPFC (medial aspect of the dorsolateral PFC). The dorsal ACC and MPFC are heavily interconnected with each other and with the posterior parietal lobe (Goldman-Rakic, 1987), reflecting their role in the spatiotemporal organization of behavior. Moreover, both regions have strong connections with the hippocampus and parahippocampal cortex (Goldman-Rakic, 1987), which may provide the anatomical basis for the observed functional coupling.

We speculate that the aforementioned mediofrontal regions contribute executive functions, such as working memory and attentional control, to regularity extraction. Specifically, we envisage that similarity detection will occur in coupled hippocampo-cortical networks through interactions of externally presented associations with stored ones (see Kumaran and McClelland, 2012 for a model of hippocampal similarity detection). The mediofrontal regions may orient attention and bias representations in these networks towards the overlapping components of memories. The concurrent activation of regularly associated face and location features in the network may then promote the linking thereof. As the fusiform gyrus and posterior parietal areas, in which facial features and locations are represented respectively, are not directly interconnected, synaptic strengthening would most likely occur through hierarchically upstream areas, including the prefrontal cortex, angular gyrus and hippocampal formation. While the hippocampus would incorporate the regular association in a new episodic memory (Tulving and Markowitsch, 1998), the PFC and angular gyrus, might build up more abstract (i.e. context poor) representations of the association, with the frontal areas including links to behavioral strategies regarding task performance. During retrieval, the $\mathrm{ACC} / \mathrm{mPFC}$ may thus function as a secondary, or additional hub, interlinking stimulus features that showed a relatively stable relation across episodes. Through this route, facial features involved in regular face-location contingencies may activate PFC-mediated general knowledge. Higher ACC/mPFC to FFA coupling over time for rule-based than for non-rule based associations supports this notion. This suggests that, with the passage of time, retrieval of rule-based associations increasingly involves a 'direct' route between the FFA and PFC. As explained earlier, it should be considered that, even though the FFA to hippocampal route appears to become less important over time, the suggested FFA to PFC route may to some extent be supplementary thereto.

A few recent studies offer tangential support for our findings. For instance, coupling between the hippocampus and the MPFC during performance on a weather prediction task, was shown to be related to the emergence of new conceptual information (Kumaran et al., 2009). In another study, using a transitive inference design, increased hippocampus-mPFC coupling tracked successful inferential memory performance (Zeithamova and Preston, 2010). Finally, a study in rats showed increased theta coherence between the hippocampus and $\mathrm{MPFC}^{3}$ following the acquisition of response rules in a maze task (Benchenane et al., 2010). Although these studies mainly regard simple stimulus-response contingencies, they do provide evidence for hippocampo-prefrontal communication in the extraction of information across multiple episodes.

Importantly, in the aforementioned study by Benchenane et al. (2010), the mPFC cell assemblies that emerged during the hippocampus-mPFC coherence state were preferentially replayed during subsequent sleep, concurrent with hippocampal sharp waves (Peyrache et al., 2009). This supports the notion that rules or regularities extracted across experiences preferentially access system-level consolidation and that such consolidation may occur to an important extent during sleep. The latter is in line with several studies showing benefits of sleep for retention (Diekelmann et al. 2009; Durrant et al., 2012; Ellenbogen et al., 2007; Sterpenich et al., 2009; Talamini et al., 2008).

Interestingly, longer-term studies (considering retention over 1 or more weeks) also implicate the ACC and MPFC in remote memory retrieval, but in co-occurrence with hippocampal disengagement (Frankland et al., 2004; Restivo et al., 2009; Takashima et al., 2006; Takehara et al., 2003). These studies suggest that mediofrontal regions may not only play a role in the formation of general knowledge, but also eventually achieve some hippocampus-independent role in its representation. In line with our earlier statement, such a role might be viewed in terms of a cortical hub, interlinking memory representations in other brain areas, which have shown a stable association over time that is relevant to behavioral guidance (for related views on hub function in system-level consolidation see Battaglia and Pennartz, 2011; Kroes and Fernández, 2012; Nieuwenhuis and Takashima, 2011; Talamini and Gorree, 2012; Van Kesteren et al., 2012).

Finally, several aspects of our design merit consideration. First, our design yielded clear effects of regularity extraction on memory performance: correct locations were chosen faster, and with more confidence, for the rule faces than the no-rule faces. Therefore, one should take into consideration that neural differences might directly reflect quantitative differences in memory strength rather than qualitatively different retrieval networks. However, this notion is rendered unlikely by the different patterning of the behavioral and neural correlates of regularity extraction across time. Indeed, our main findings at the neural level either regard increasing differences across time, or differences at the remote time only. On the other hand, confidence and RT differences between conditions, which were already apparent at the immediate time

\footnotetext{
${ }^{3}$ In the rodent literature, the term $\mathrm{MPFC}$ refers to a frontal region that has functions that are similar to those of the dorsolateral and possibly medial regions in the primate frontal lobe. The rat mPFC encompasses several frontal areas on the medial aspect of the rostral cortex, including the ACC (Bryan and Cioe, 2004).
} 
point, remained stable or decreased across time. Furthermore, one might expect differences in encoding strength to be reflected in different activity levels in retrieval areas, which were not observed. A causal relation between these behavioral effects and the observed connectivity differences therefore seems implausible, although it cannot be excluded.

Secondly, since the current design investigated brain activity and connectivity at different time points, it should be taken into account that non-specific differences across scan sessions might have partially influenced the results. However, this only relates to the main effects of time, where recent and remote conditions were directly compared. Effects of regularities on connectivity data either regarded an interaction or only considered the remote time point. Hence, our primary results on differential consolidation mechanisms for rule and no-rule faces do not suffer from this potential limitation.

Lastly, we would like to acknowledge various models concerning generalization, rule and category learning that are pertinent to our account. First, neural network accounts considering complementary learning systems in the hippocampal and neocortical areas, have shown how interplay of these systems can lead to the formation of generalized cortical representations that capture the common structure in ensembles of experiences (McClelland and Goddard, 1996; McClelland et al., 1995). Our data offer some first insights into how the global processes proposed in these models are implemented in actual brain circuits. Second, several mathematical models describe putative processes underlying category formation in a neurally agnostic manner (Ashby, 1992; Ashby and O'Brien, 2005; Maddox and Ashby, 1993; Minda and Smith, 2002; Nosofsky and Zaki, 2002; Nosofsky et al., 1994). For example, exemplar models propose that all unique experiences are stored in separate memory traces (Kruschke, 1992; Nosofsky, 1986). Deciding to which category a new exemplar belongs, results from calculating its similarity to all stored exemplars. Prototype models (Nosofsky and Zaki, 2002; Rosch, 2002) on the other hand, suggest that we form summary representations that typify categories. Although the present data is by no means suitable to dissociate between these individual models, our interpretations are slightly more in line with prototype models, as we propose abstract representations might be formed through the extraction of regularities across event memories.

\section{Conclusion}

As memories age, they appear to lose contextual richness and instead, acquire a more factual, semantic nature. According to a recent study, this occurs through preferential loss of hippocampus-dependent configurational components from memory, as of one week after encoding (Talamini and Gorree, 2012). Other studies have also shown diminished context-sensitivity of memories over time (Zhou and Riccio, 1996), some of them in relation to hippocampal disengagement from retrieval (Wiltgen and Silva, 2007; Winocur et al., 2007). This suggests that system-level consolidation is related to a loss of memories' configurational complexity. Indeed, many, if not most, hippocampusencoded configurations may be lost with degradation of the hippocampal trace, whereas only a fraction is retained through system-level consolidation. Our findings suggest that one factor determining which configurations are retained, relates to their stability across episodes. These findings provide some first insights into the poorly understood interplay between episodic and semantic memory, suggesting that the consolidation of cross-episodic regularities may constitute a key mechanism underlying general knowledge acquisition.

\section{Acknowledgments}

We thank Max Keuken for valuable suggestions and comments on the manuscript, Paul Gaalman for technical assistance, and the Netherlands Organisation for Scientific Research (NWO) for funding via a VIDI grant (grant number C.2523.0126.01).

\section{Appendix A. Supplementary data}

Supplementary data to this article can be found online at http://dx. doi.org/10.1016/j.neuroimage.2013.10.063.

\section{References}

Ashby, F.G., 1992. Multidimensional models of categorization. Multidimensional Models of Perception and Cognition.

Ashby, F.G., O'Brien, J.B., 2005. Category learning and multiple memory systems. Trends Cogn. Sci. 9, 83-89.

Battaglia, F.P., Pennartz, C.M.A., 2011. The construction of semantic memory: grammarbased representations learned from relational episodic information. Front. Comput. Neurosci. 5, 22.

Bayley, P.J., Squire, L.R., 2005. Failure to acquire new semantic knowledge in patients with large medial temporal lobe lesions. Hippocampus 15, 273-280.

Bayley, P.J., Hopkins, R.O., Squire, L.R., 2006. The fate of old memories after medial temporal lobe damage. J. Neurosci. 26, 13311-13317.

Benchenane, K., Peyrache, A., Khamassi, M., Tierney, P.L., Gioanni, Y., Battaglia, F.P., Wiener, S.I., 2010. Coherent theta oscillations and reorganization of spike timing in the hippocampal-prefrontal network upon learning. Neuron 66, 921-936.

Binder, J.R., Desai, R.H., Graves, W.W., Conant, L.L., 2009. Where is the semantic system? A critical review and meta-analysis of 120 functional neuroimaging studies. Cereb. Cortex 19, 2767-2796.

Birrell, J.M., Brown, V.J., 2000. Medial frontal cortex mediates perceptual attentional set shifting in the rat. J. Neurosci. 20, 4320-4324

Blumenfeld, R.S., Ranganath, C., 2007. Prefrontal cortex and long-term memory encoding: an integrative review of findings from neuropsychology and neuroimaging. Neuroscientist 13, 280-291.

Brown, A.S., 2002. Consolidation theory and retrograde amnesia in humans. Psychon. Bull. Rev. 9, 403-425.

Bryan, K., Cioe, J., 2004. Organization and plasticity of the prefrontal cortex of the rat prefrontal cortex. In: Otani, S. (Ed.), From Synaptic Plasticity to Cognition. Springer, pp. 1-32.

Burgess, N., Becker, S., King. J.A., O'Keefe, J., 2001. Memory for events and their spatial context: models and experiments. Philos. Trans. R. Soc. Lond. B Biol. Sci. 356, 1493-1503.

Burgess, N., Maguire, E.A., O'Keefe, J., 2002. The human hippocampus and spatial and episodic memory. Neuron 35, 625-641.

Diekelmann, S., Wilhelm, I., Born, J., 2009. The whats and whens of sleep-dependent memory consolidation. Sleep Med. Rev. 13, 309-321.

Drewe, E.A., 1974. The effect of type and area of brain lesion on Wisconsin card sorting test performance. Cortex 10, 159-170.

Durrant, S.J., Cairney, S.A., Lewis, P.A., 2012. Overnight consolidation aids the transfer of statistical knowledge from the medial temporal lobe to the striatum. Cereb. Cortex $1-12$.

Ellenbogen, J.M., Hu, P.T., Payne, J.D., Titone, D., Walker, M.P., 2007. Human relational memory requires time and sleep. Proc. Natl. Acad. Sci. U. S. A. 104, 7723-7728.

Frankland, P.W., Bontempi, B., Talton, L.E., Kaczmarek, L., Silva, A.J., 2004. The involvement of the anterior cingulate cortex in remote contextual fear memory. Science 304 , 881-883.

Friston, K., Buechel, C., Fink, G., Morris, J., Rolls, E., Dolan, R., 1997. Psychophysiological and modulatory interactions in neuroimaging. NeuroImage 6, 218-229.

Gardiner, J.M., Brandt, K.R., Baddeley, A.D., Vargha-Khadem, F., Mishkin, M., 2008. Charting the acquisition of semantic knowledge in a case of developmental amnesia. Neuropsychologia 46, 2865-2868.

Gauthier, I., Tarr, M.J., Moylan, J., Skudlarski, P., Gore, J.C., Anderson, A.W., 2000. The fusiform "face area" is part of a network that processes faces at the individual level. J. Cogn. Neurosci. 12, 495-504.

Goldman-Rakic, P.S., 1987. Circuitry of primate prefrontal cortex and regulation of behavior by representational memory. In: Plum, F., Mountcastle, V. (Eds.), Handbook of Physiology: The Nervous System. American Physiological Society, pp. 373-417.

Grill-Spector, K., Knouf, N., Kanwisher, N., 2004. The fusiform face area subserves face perception, not generic within-category identification. Nat. Neurosci. 7, 555-562.

Hayasaka, S., Nichols, T.E., 2003. Validating cluster size inference: random field and permutation methods. NeuroImage 20, 2343-2356.

Hayman, C.A.G., Macdonald, C.A., Tulving, E., 1993. The role of repetition and associative interference in new semantic learning in amnesia: a case experiment. J. Cogn. Neurosci. 5, 375-389.

IQ Biometrix, 2003. FACES, the Ultimate Composite Picture.

Kanwisher, N., McDermott, J., Chun, M., 1997. The fusiform face area: a module in human extrastriate cortex specialized for face perception. J. Neurosci. 17, 4302-4311.

Kesner, R.P., 2009. The posterior parietal cortex and long-term memory representation of spatial information. Neurobiol. Learn. Mem. 91, 197-206.

Kroes, M.C.W., Fernández, G., 2012. Dynamic neural systems enable adaptive, flexible memories. Neurosci. Biobehav. Rev. 36, 1646-1666.

Kruschke, J.K., 1992. Chapter 18 ALCOVE: an exemplar-based connectionist model of category learning. Psychol. Rev. 99, 22-44.

Kumaran, D., McClelland, J.L., 2012. Generalization through the recurrent interaction of episodic memories: a model of the hippocampal system. Psychol. Rev. 119, 573-616.

Kumaran, D., Summerfield, J.J., Hassabis, D., Maguire, E.A., 2009. Tracking the emergence of conceptual knowledge during human decision making. Neuron 63, 889-901.

Lisman, J.E., Talamini, L.M., Raffone, A., 2005. Recall of memory sequences by interaction of the dentate and CA3: a revised model of the phase precession. Neural Netw. 18, $1191-1201$ 
Maddox, W.T., Ashby, F.G., 1993. Comparing decision bound and exemplar models of categorization. Percept. Psychophys. 53, 49-70.

Manns, J.R., Hopkins, R.O., Squire, L.R., 2003. Semantic memory and the human hippocampus. Neuron 38, 127-133.

Maren, S., Aharonov, G., Fanselow, M.S., 1997. Neurotoxic lesions of the dorsal hippocampus and Pavlovian fear conditioning in rats. Behav. Brain Res. 88, 261-274.

Martin, A., Chao, L.L., 2001. Semantic memory and the brain. Curr. Opin. Neurobiol. 11, 194-201.

Maviel, T., Durkin, T.P., Menzaghi, F., Bontempi, B., 2004. Sites of neocortical reorganization critical for remote spatial memory. Science 305, 96-99.

McClelland, J.L., Goddard, N.H., 1996. Considerations arising from a complementary learning systems perspective on hippocampus and neocortex. Hippocampus 6, 654-665.

McClelland, J.L., McNaughton, B.L., O'Reilly, R.C., 1995. Why there are complementary learning systems in the hippocampus and neocortex: insights from the successes and failures of connectionist models of learning and memory. Psychol. Rev. 102, 419-457.

Meeter, M., Murre, J.M.J., 2005. Tracelink: a model of consolidation and amnesia. Cogn. Neuropsychol. 22, 559-587.

Meeter, M., Murre, J.M.J., Talamini, L.M., 2004. Mode shifting between storage and recall based on novelty detection in oscillating hippocampal circuits. Hippocampus 14 , 722-741.

Minda, J.P., Smith, J.D., 2002. Comparing prototype-based and exemplar-based accounts of category learning and attentional allocation. J. Exp. Psychol. Learn. Mem. Cogn. 28, 275-292.

Mishkin, M., 1978. Memory in monkeys severely impaired by combined but not by separate removal of amygdala and hippocampus. Nature 273, 297-298.

Moscovitch, M., Rosenbaum, R.S., Gilboa, A., Addis, D.R., Westmacott, R., Grady, C. McAndrews, M.P., Levine, B., Black, S., Winocur, G., Nadel, L., 2005. Functional neuroanatomy of remote episodic, semantic and spatial memory: a unified account based on multiple trace theory. J. Anat. 207, 35-66.

Murre, J.M.J., Meeter, M., Chessa, A.G., 2006. Modeling amnesia: connectionist and mathematical approaches. Statistical and Process Models for Cognitive Neuroscience and Aging. Psychology Press.

Nadel, L., Moscovitch, M., 1997. Memory consolidation, retrograde amnesia and the hippocampal complex. Curr. Opin. Neurobiol. 7, 217-227.

Nieuwenhuis, I.L.C., Takashima, A., 2011. The role of the ventromedial prefrontal cortex in memory consolidation. Behav. Brain Res. 218, 325-334.

Nosofsky, R.M., 1986. Attention, similarity, and the identification-categorization relationship. J. Exp. Psychol. Gen. 115, 39-61.

Nosofsky, R.M., Zaki, S.R., 2002. Exemplar and prototype models revisited: response strategies, selective attention, and stimulus generalization. J. Exp. Psychol. Learn. Mem. Cogn. 28, 924-940.

Nosofsky, R.M., Gluck, M.A., Palmeri, T.J., McKinley, S.C., Glauthier, P., 1994. Comparing models of rule-based classification learning: a replication and extension of Shepard, Hovland, and Jenkins (1961). Mem. Cogn. 22, 352-369.

Otani, S., 2004. Prefrontal Cortex; From Synaptic Plasticity to Cognition. Springer.

Patterson, K., Nestor, P.J., Rogers, T.T., 2007. Where do you know what you know? The representation of semantic knowledge in the human brain. Nature reviews. Neuroscience 8, 976-987.

Peyrache, A., Khamassi, M., Benchenane, K., Wiener, S.I., Battaglia, F.P., 2009. Replay of rule-learning related neural patterns in the prefrontal cortex during sleep. Nat. Neurosci. 12, 919-926.

Piefke, M., 2003. Differential remoteness and emotional tone modulate the neural correlates of autobiographical memory. Brain 126, 650-668.

Quillfeldt, J.A., Zanatta, M.S., Schmitz, P.K., Quevedo, J., Schaeffer, E., Lima, J.B., Medina, J.H., Izquierdo, I., 1996. Different brain areas are involved in memory expression at different times from training. Neurobiol. Learn. Mem. 66, 97-101.

Restivo, L., Vetere, G., Bontempi, B., Ammassari-Teule, M., 2009. The formation of recent and remote memory is associated with time-dependent formation of dendritic spines in the hippocampus and anterior cingulate cortex. J. Neurosci. 29, 8206-8214.

Rosch, E., 2002. Principles of categorization. In: Levitin, D.J. (Ed.), Foundations of Cognitive Psychology Core Readings. MIT Press, pp. 251-270.
Rosenbaum, R.S., Winocur, G., Moscovitch, M., 2001. New views on old memories: reevaluating the role of the hippocampal complex. Behav. Brain Res. 127, 183-197.

Sack, A.T., 2009. Parietal cortex and spatial cognition. Behav. Brain Res. 202, 153-161.

Scoville, W.B., Milner, B., 1957. Loss of recent memory after bilateral hippocampal lesions. J. Neurol. Neurosurg. Psychiatry 20, 11-21.

Squire, L.R., 1992. Memory and the hippocampus: a synthesis from findings with rats, monkeys, and humans. Psychol. Rev. 99, 195-231.

Squire, L.R., Slater, P.C., Chace, P.M., 1975. Retrograde amnesia: temporal gradient in very long term memory following electroconvulsive therapy. Science 187, 77-79.

Sterpenich, V., Albouy, G., Darsaud, A., Schmidt, C., Vandewalle, G., Dang Vu, T.T., Desseilles, M., Phillips, C., Degueldre, C., Balteau, E., Collette, F., Luxen, A., Maquet, P., 2009. Sleep promotes the neural reorganization of remote emotional memory. J. Neurosci. Off. J. Soc. Neurosci. 29, 5143-5152.

Swanson, L.W., 1981. A direct projection from Ammon's horn to prefrontal cortex in the rat. Brain Res. 217, 150-154.

Takashima, A., Petersson, K.M., Rutters, F., Tendolkar, I., Jensen, O., Zwarts, M.J. McNaughton, B.L., Fernández, G., 2006. Declarative memory consolidation in humans: a prospective functional magnetic resonance imaging study. Proc. Natl. Acad. Sci. U. S. A 103, 756-761.

Takashima, A., Nieuwenhuis, I.L.C., Rijpkema, M., Petersson, K.M., Jensen, O., Fernández, G. 2007. Memory trace stabilization leads to large-scale changes in the retrieval network: a functional MRI study on associative memory. Learn. Mem. 14, 472-479.

Takashima, A., Nieuwenhuis, I.L.C., Jensen, O., Talamini, L.M., Rijpkema, M., Fernández, G., 2009. Shift from hippocampal to neocortical centered retrieval network with consolidation. J. Neurosci. 29, 10087-10093.

Takehara, K., Kawahara, S., Takatsuki, K., Kirino, Y., 2002. Time-limited role of the hippocampus in the memory for trace eyeblink conditioning in mice. Brain Res. 951, 183-190.

Takehara, K., Kawahara, S., Kirino, Y., 2003. Time-dependent reorganization of the brain components underlying memory retention in trace eyeblink conditioning. J. Neurosci. 23, 9897-9905.

Talamini, L.M., Gorree, E., 2012. Aging memories: differential decay of episodic memory components. Learn. Mem. 19, 239-246.

Talamini, L.M., Nieuwenhuis, I.L.C., Takashima, A., Jensen, O., 2008. Sleep directly following learning benefits consolidation of spatial associative memory. Learn. Mem. 15, 233-237.

Teng, E., Squire, L.R., 1999. Memory for places learned long ago is intact after hippocampal damage. Nature 400, 675-677.

Thierry, A.M., Gioanni, Y., Dégénétais, E., Glowinski, J., 2000. Hippocampo-prefrontal cortex pathway: anatomical and electrophysiological characteristics. Hippocampus 10, 411-419.

Tulving, E., 1983. Elements of Episodic Memory. Clarendon, Oxford.

Tulving, E., 1985. How many memory systems are there? Am. Psychol. 40, 385-398.

Tulving, E., Markowitsch, H.J., 1998. Episodic and declarative memory: role of the hippocampus. Hippocampus 8, 198-204.

Van Kesteren, M.T.R., Fernández, G., Norris, D.G., Hermans, E.J., 2010. Persistent schemadependent hippocampal-neocortical connectivity during memory encoding and postencoding rest in humans. Proc. Natl. Acad. Sci. U. S. A. 107, 7550-7555.

Van Kesteren, M.T.R., Ruiter, D.J., Fernández, G., Henson, R.N., 2012. How schema and novelty augment memory formation. Trends Neurosci. 35, 211-219.

Wiltgen, B.J., Silva, A.J., 2007. Memory for context becomes less specific with time. Learn. Mem. 14, 313-317.

Winocur, G., Moscovitch, M., Sekeres, M., 2007. Memory consolidation or transformation: context manipulation and hippocampal representations of memory. Nat. Neurosci. $10,555-557$.

Zeithamova, D., Preston, A.R., 2010. Flexible memories: differential roles for medial temporal lobe and prefrontal cortex in cross-episode binding. J. Neurosci. 30 $14676-14684$.

Zhou, Y., Riccio, D., 1996. Manipulation of components of context: the context shift effect and forgetting of stimulus attributes. Learn. Motiv. 27, 400-407.

Zola-Morgan, S., Squire, L.R., 1985. Medial temporal lesions in monkeys impair memory on a variety of tasks sensitive to human amnesia. Behav. Neurosci. 99, 22-34. 